\title{
44 Pancreatic Exocrine Dysfunction
}

\author{
DAVID WHITCOMB AND JONATHAN COHN
}

\section{INTRODUCTION}

The pancreas is a retroperitoneal gland with both endocrine and exocrine functions. Pancreatic endocrine hormones are released by the islets into the circulation where they are essential for the regulation of intermediary metabolism (see Chapter 47). Pancreatic exocrine secretions (pancreatic juice), produced by the concerted action of pancreatic acinar and ductal cells, are delivered into the duodenum where they are essential for normal digestion. Different pancreatic enzymes promote the efficient digestion of complex carbohydrates, lipids, proteins, and other nutrients (Table 44-1). It is noteworthy that many of these digestive enzymes have the potential to digest components of the pancreas itself. Such autodigestion is normally prevented by several mechanisms. For example, many digestive enzymes exist in an inactive (proenzyme) form until they reach the duodenum. Other mechanisms either prevent activation of proenzymes inside the pancreas or inhibit the enzymes if they are inadvertently activated prematurely.

Pancreatic exocrine dysfunction can present clinically either as an acute illness (acute pancreatitis) or as a chronic process (pancreatic insufficiency with or without chronic pancreatitis). Inherited causes of pancreatic exocrine disease include hereditary pancreatitis and cystic fibrosis (CF). In both conditions, the responsible gene is now known and information is rapidly emerging concerning the role of the gene's protein product during normal pancreatic function and during the pathogenesis of pancreatic exocrine dysfunction.

\section{ACUTE PANCREATITIS}

BACKGROUND Acute pancreatitis is defined clinically as pancreatic inflammation characterized by the sudden onset of abdominal pain in association with elevation in blood or urine pancreatic enzyme levels. In about $80 \%$ of the cases, acute pancreatitis is mild and recovery is expected. However, in the remaining cases, acute pancreatitis is severe and can produce potentially lifethreatening complications. The keys to clinical management are early recognition of severe acute pancreatitis, anticipation of complications, and prevention of recurrence.

CLINICAL FEATURES Acute pancreatitis usually causes epigastric abdominal pain of acute onset. The pain tends to be constant and may radiate to the mid-back. Vomiting occurs in $80 \%$ of cases and provides little pain relief. Abdominal tenderness with guarding

From: Principles of Molecular Medicine (J. L. Jameson, ed.), (C1998 Humana Press Inc., Totowa, NJ. occurs in half of cases but tends to be less severe than anticipated based on either the severity of reported pain or the degree of pancreatic inflammation (by abdominal CT or ultrasound), and this discrepancy results from the retroperitoneal location of the pancreas.

DIAGNOSIS In a patient presenting with typical abdominal pain, a serum amylase or lipase level more then 2.5 times the upper limit of normal strongly suggests the diagnosis of acute pancreatitis. CT with intravenous contrast is the most sensitive method of assessing the degree of pancreatic injury, pancreatic necrosis, and local extrapancreatic complications but is usually unnecessary in mild pancreatitis. In most cases, acute pancreatitis is associated with an identifiable cause, with gallstones and alcohol ingestion being the most common factors (Table 44-2). When the cause of pancreatitis is identified early, this may suggest strategies to prevent ongoing pancreatic injury and to prevent recurrence.

MOLECULAR PATHOPHYSIOLOGY A breakthrough in understanding the nature of acute pancreatitis came in 1896 when Chiari proposed that this condition represents autodigestion, rather than infection, of the pancreas. Autodigestion of the pancreas is a major threat because this organ normally produces enormous quantities of potentially dangerous digestive enzymes. To prevent autodigestion, three types of protective strategies are employed. In the first strategy, many enzymes (except amylase and lipase) are synthesized as proenzymes requiring enzymatic cleavage for activation. Activation normally occurs after the proenzymes are secreted by the pancreas into the intestinal lumen, where the intestinal brush border enzyme, enterokinase, cleaves trypsinogen activation peptide (TAP) from trypsinogen (the proenzyme) to generate trypsin (the active enzyme). Trypsin also activates trypsinogen and efficiently activates all other pancreatic digestive enzymes. Thus, the activation of trypsin in the duodenum is highly desirable as a key element in the rapid propagation of the digestive enzyme activation cascade.

In the pancreas, however, widespread activation of trypsin may be disastrous. This is especially important in humans because human trypsinogen has a propensity to autoactivate. Additional protective strategies are therefore necessary to prevent autoactivation of the digestive enzyme cascade in the pancreas. For example, within the acinar cell, autoactivation of trypsinogen and other digestive enzymes is limited by maintenance of low intracellular calcium, by maintenance of a neutral $\mathrm{pH}$, and by the subcellular isolation of zymogen granules. Furthermore, because of a constant background of trypsinogen autoactivation, a limited amount of pancreatic secretory trypsin inhibitor (PSTI) is synthe- 
Table 44-1

Pancreatic Digestive Enzymes

Proteinases

Trypsin(ogen),

Chymotrypsin(ogen),

(Pro)carboxypeptidases,

(Pro)elastases

Kalikreinogen

Pancreatic $\alpha$-amylase

Pancreatic lipase

Other enzymes

(Pro)colipase I, II

(Pro)Phospholipase A2

Ribonuclease

Deoxyribonuclease I sized with trypsinogen. If trypsin activity in the acinar cells exceeds the inhibitory capacity of PSTI, then a trypsin self-destruct mechanism is initiated in which the peptide chain connecting the two globular domains of trypsin is cut at arganine II (R117) by trypsin and mesotrypsin. Cleavage at this site permanently inactivates trypsin and other digestive enzymes with this design because the two halves of the molecule separate, thereby eliminating the active site of the enzyme. Finally, if active digestive enzymes escape from the pancreatic acinar cells into the intracellular space or plasma, they are inactivated by hepatocyte-derived $\alpha_{2}$-macroglobulin and $\alpha_{1}$-protease inhibitors. Thus, multiple protective strategies exist to prevent pancreatic autodigestion and acute pancreatitis.

The sequence of events initiating acute pancreatitis at the cellular level remains controversial, but several features are common to most models of experimental pancreatitis. Microscopically, zymogen granules accumulate at the apex of the acinar cells without being secreted. This functional block of secretion reflects the disruption of normal intracellular mechanisms, including the organization of microtubules and other proteins, which are necessary for exocytosis. The second feature is premature activation of digestive enzymes within the acinar cells. Although the intracellular site and mechanism responsible for digestive enzyme activation is uncertain, it seems clear that activation of these enzymes promotes "autodigestion" of pancreatic cells and initiates an inflammatory reaction.

Once an episode of acute pancreatitis is triggered, a characteristic inflammatory reaction occurs that is independent of the initiating factors. When acinar cells are damaged, they begin leaking activated digestive enzymes and other cellular elements into the interstitial space. This triggers an inflammatory response with the release of proinflammatory mediators, including interleukin-1 (IL-1), IL-2, IL-6, IL-8, tumor necrosis factor- $\alpha$ (TNF- $\alpha$ ), and platelet-activating factor (PAF) (see Chapter 30). If pancreatic injury is mild, then the inflammatory response is self-limited and the process contained. However, if pancreatic injury is severe, then a more intense inflammatory response ensues. This inflammatory response is responsible for many of the local and systemic complications of acute pancreatitis. For example, the acute phase reaction and hypermetabolic state in severe acute pancreatitis with fever, decreased peripheral vascular resistance, and increased cardiac output are driven by cytokines. The pulmonary complications of hypoxia and adult respiratory distress syndrome (ARDS) largely develop because of the release of phospholipase $A_{2}$ from activated macrophages. Finally, the progression of pancreatic edema to
Table 44-2

Causes of Acute Pancreatitis

Mechanical

Gallstones

Biliary microlithiasis

Post ERCP (multifactorial)

Pancreatic cancer

Anatomical predisposition

Pancreas divisum

Annular pancreas

? sphincter of Oddi dysfunction

Toxins

Chronic alcohol abuse (multifactorial)

Scorpion bite

Medications

Anticholinesterases

$L$-asparaginase

Azathioprine

Dideoxyinosine (DDI)

Estrogen

6-Mercaptopurine

Salicylates

Thiazide-diuretics

Valproic acid

Vinca alkaloids

Other (idiosyncratic reactions)

Hereditary Trypsinogen gene mutation (? others)

Metabolic Hyperlipidemia (triglycerides $>1000$ )

Hypercalcemia

Trauma Blunt abdominal trauma

Penetrating ulcer

Postoperative/post-ERCP

Infections Mycoplasma pneumonia

Virus (mumps, coxsackie, CMV, HSV, HIV)

Bacteria

Mycobacterium avium-intracellular

Vascular Vasculitis (e.g., connective tissue diseases)

Hypotension (Postoperative: e.g., CABG)

Infarction

Idiopathic Unknown

pancreatic necrosis may be driven, in part, by TNF- $\alpha$. In animal models of acute pancreatitis, when proinflammatory cytokines are inhibited (i.e., PAF inhibitors) or the anti-inflammatory cytokine IL-10 is given, the severity of pancreatic injury is reduced, and survival improves. Although clinical intervention at the initiation of acute pancreatitis is impossible in most cases, the severity of acute pancreatitis may be reduced by early administration of agents that modify the immune response. Specific PAF inhibitors, for example, show great promise in this regard. Thus, acute pancreatitis could be considered to progress through three general phases: an acute injury from a variety of causes, a characteristic inflammatory response that depends on the severity of injury, followed by a healing phase.

GENETIC PREDISPOSITION TO ACUTE PANCREATITIS Hereditary pancreatitis and familial hypertryglyceridemia predispose affected family members to acute pancreatitis. Hereditary pancreatitis represents a primary disorder of the pancreas, whereas patients with familial hypertryglyceridemia suffer from pancreatitis secondary to markedly elevated tryglycerides. Hereditary pancreatitis is a chronic, recurrent inflammatory disorder of the pancreas affecting family members in multiple generations. This disorder encompasses all features of acute pancreatitis and chronic pancreatitis. Thus, understanding the molecular basis 
of pancreatic injury in hereditary pancreatitis may provide insight into the pathogenesis of other forms of acute and chronic pancreatitis.

Hereditary pancreatitis is inherited as an autosomal dominant trait with $80 \%$ penetration and variable expression. Nearly 100 kindreds have been reported since the genetic nature of this disorder was recognized by Comfort and Steinberg in 1952. The cause of hereditary pancreatitis was determined after the gene was localized to chromosome VII and then identified by sequencing of candidate genes. This analysis showed that hereditary pancreatitis is caused by a mutation that was in a cationic trypsinogen gene, resulting in an arginine to histidine ( $\mathrm{R}-\mathrm{H})$ substitution at residue 117. This $\mathrm{R} 117 \mathrm{H}$ mutation was observed in all patients with hereditary pancreatitis and in all obligate carriers from five kindreds, but not in individuals who married into the families nor in 140 unrelated individuals.

The effect of the $\mathrm{R} 117 \mathrm{H}$ mutation on trypsinogen regulation has been studied through the X-ray crystal structure, molecular modeling, and protein digest analyses. Taken together, these data suggest that R117 occurs in a trypsin-sensitive site in the chain connecting the two globular domains of trypsinogen, and that the $\mathrm{R} 117 \mathrm{H}$ mutation eliminates the final control of excessive trypsin activity in the pancreas by removing the natural cleavage site of the trypsin-like enzymes (Fig. 44-1). This finding explains why hereditary pancreatitis is an autosomal-dominant disorder. Even if only half of the trypsinogen molecules resist proteolytic degradation, this is sufficient to initiate the digestive enzyme activation cascade in the pancreas and therefore express the phenotype. This also explains why attacks of acute pancreatitis occur episodically rather than continually: Attacks occur when the rate of intrapancreatic trypsinogen activation overwhelms the protective effects of PSTI.

The discovery of the hereditary pancreatitis gene has had important implications for understanding the pathogenesis of pancreatitis in general. First, it showed that intrapancreatic protease activation is sufficient to cause acute pancreatitis in humans. Second, it emphasizes the importance of trypsinogen autoactivation in humans and may explain why it has been difficult to study acute pancreatitis in animal models in which trypsinogen is more stable. Finally, it demonstrates a direct link between recurrent acute pancreatitis and chronic pancreatitis.

MANAGEMENT AND TREATMENT Mild pancreatitis tends to be a self-limited process. Treatment is supportive and includes pancreatic rest (nothing by mouth), intravenous hydration, and analgesics. Patients usually recover within 3 days and can be discharged from the hospital when oral intake is adequate to prevent dehydration.

By contrast, severe pancreatitis can result in life-threatening complications requiring aggressive management. Because these patients are at risk for sudden death, ICU admission is often indicated during the initial phase of severe acute pancreatitis. To predict which patients with acute pancreatitis are most likely to develop life-threatening complications, several prognostic schemes have been developed (see Table 44-3). These schemes remain useful for assisting in identification of the subset of patient with acute pancreatitis for whom intensive monitoring is most appropriate.

Early recognition is key to the management of the most common life-threatening complications of severe acute pancreatitis. Cardiovascular collapse and respiratory failure each may occur rapidly and require aggressive support. Multiorgan failure may develop as nutritional stores are depleted, and early nutritional support should

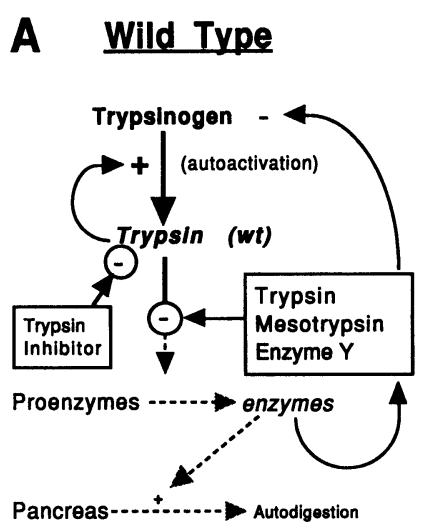

\section{B HP Mutation}

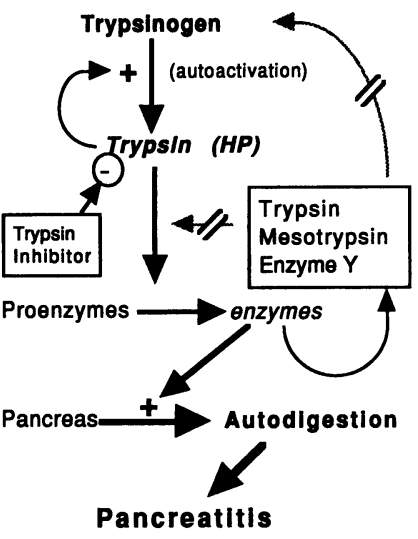

Figure 44-1 Model of trypsin self-destruct mechanism preventing pancreatic autodigestion. (A) Autoactivation and enzymatic activation of trypsinogen generate trace amounts of active trypsin within pancreatic acinar cells. Active trypsin is inhibited by a limited supply of trypsin inhibitor (PSTI). If trypsin activity exceeds the inhibitory capacity of PSTI, then proenzymes, including mesotrypsin and enzyme Y, are activated. These enzymes feed-back to inactivate wildtype (wt) trypsinogen, trypsin, and other zymogens. (B) Activation of mutant (HP) trypsin in amounts that exceed the inhibitory capacity of PSTI results in activation of proenzymes. Since the Arg 117 cleavage site for trypsin-like enzymes is replaced by His in the HP mutant trypsin, trypsin continues to activate trypsinogen and other zymogens unabated, leading to autodigestion of the pancreas and pancreatitis.

Table 44-3

\section{Prognostic Criteria in Acute Pancreatitis}

\begin{tabular}{ll}
\hline Ranson Criteria $^{a}$ & On admission \\
& Age $>55$ years \\
& Leukocyte count $>16,000 / \mathrm{mm}^{3}$ \\
& Blood glucose $>200 \mathrm{mg} / \mathrm{dL}$ \\
& Serum LDH $>350 \mathrm{IU} / \mathrm{L}$ \\
& Serum GOT (SGOT/AST) $>250 \mathrm{IU} / \mathrm{dL}$ \\
& At 48 h Hematocrit decreases $>10 \%$ \\
& BUN rises $>5 \mathrm{mg} / \mathrm{dL}$ \\
& Serum calcium $<8 \mathrm{mg} / \mathrm{dL}$ \\
& Arterial PO2 $<60 \mathrm{mmHg}$ \\
& Base deficit $>4 \mathrm{mEq} / \mathrm{L}$ \\
& Estimated fluid sequestration $>6 \mathrm{~L}$ \\
& On Admission \\
& Age $>55$ years \\
& WBC count $>15,000 / \mu \mathrm{L}$ \\
& Glucose $>180 \mathrm{mg} / \mathrm{dL}$ \\
Glasgow Criteria & \\
& BUN $>45 \mathrm{mg} / \mathrm{dL}$ \\
& PO2 $<60 \mathrm{mmHg}$ \\
& Albumin $<3.2 \mathrm{~g} / \mathrm{dL}$ \\
& Calcium $<8 \mathrm{mg} / \mathrm{dL}$ \\
& LDH $>600 \mathrm{IU} / \mathrm{L}$ \\
\hline
\end{tabular}

${ }^{a}$ Each factor is one point. Three or more points suggest severe acute pancreatitis

${ }^{b}$ Each factor is one point. Three or more points suggest severe acute pancreatitis

be considered. Sepsis and infected pancreatic necrosis also are commonly occurring complications. Prophylactic broad spectrum antibiotics such as imipenem or cefuroxime may reduce the frequency of these serious infectious complications. 
FUTURE DIRECTIONS Because the injury phase of acute pancreatitis is usually brief and early recognition is difficult, prevention is important. Understanding the sites and mechanisms of early enzyme activation, the factors controlling the inflammatory response, and the resulting pathologic events may be helpful in limiting pancreatic damage when pancreatic injury occurs. Now that the cause of hereditary pancreatitis is known and the gene has been cloned, transgene animal models can be developed to aid in the study of human pancreatitis, and strategies to prevent hereditary pancreatitis and other forms of pancreatitis can be developed.

Increasing knowledge of the cytokine networks are also important. PAF inhibitors that have been designed through computer simulations may be clinically useful in reducing the severity of severe acute pancreatitis if given within 24-48 hours. These advances offer new hope in the prevention and treatment of acute pancreatitis.

\section{PANCREATIC INSUFFICIENCY}

BACKGROUND Chronic pancreatitis differs from acute pancreatitis in that there is permanent loss of exocrine function, resulting in pancreatic insufficiency. This is often associated with persistent pancreatic inflammation, leading to fibrosis and atrophy of the pancreas and to dilatation and distortion of the ducts. In adults, the most common cause of chronic pancreatitis and of pancreatic insufficiency is alcohol. In children, CF is the most frequent cause of pancreatic insufficiency, and chronic pancreatitis rarely occurs.

CF is a common disease with an incidence of $1 / 2000$ in the United States. Roughly $90 \%$ of patients with CF develop pancreatic insufficiency, and pancreatic dysfunction is such a prominent feature of CF that the disease was initially termed fibrocystic disease of the pancreas. This section will discuss pancreatic insufficiency with an emphasis on the molecular pathogenesis of pancreatic insufficiency in CF.

CLINICAL FEATURES The most important manifestations of pancreatic insufficiency result from malabsorption of incompletely digested nutrients. Malabsorption of fat and fat-soluble vitamins is usually prominent, leading to steatorrhea associated with delayed growth or weight loss. In patients with $\mathrm{CF}$, meconium ileus or pulmonary symptoms often suggest the diagnosis before pancreatic insufficiency is evident. (The clinical features of $\mathrm{CF}$ are described further in Chapter 37.) In adults, the most common cause of pancreatic insufficiency is chronic pancreatitis resulting from alcoholism. Prominent symptoms include chronic midepigastric pain (often postprandial), weight loss (largely as a result of food avoidance), and steatorrhea. In addition, diabetes mellitus sometimes accompanies pancreatic insufficiency resulting from $\mathrm{CF}$ or advanced chronic pancreatitis.

DIAGNOSIS A diagnosis of pancreatic insufficiency is suggested by the combination of steatorrhea (malabsorption of more than $5 \%$ of ingested fat during a 24 - or 72 -h stool collection) with normal bowel mucosal morphology (by biopsy) and function (e.g., D-xylose absorption). The diagnosis is supported by a clear response to pancreatic enzyme replacement therapy. When the diagnosis is uncertain, the reference standard for documenting pancreatic insufficiency is the secretin test. In this test, the duodenum is intubated to measure the amount of $\mathrm{HCO}_{3}{ }^{-}$and fluid secreted by the pancreas in response to secretin. Pancreatic insufficiency can also be detected by noninvasive tests such as the duallabel Schilling test, benteromide test, or Sudan stain.
In children and young adults with pancreatic insufficiency, a diagnosis of CF is most commonly established by the sweat test (quantitative pilocarpine iontophoresis). When a child with pancreatic insufficiency has a normal sweat test, the differential diagnosis includes Shwachman's syndrome (an autosomal recessive condition associated with neutropenia) and rare enzyme deficiencies (e.g., congenital lipase deficiency).

In adults, pancreatic insufficiency most often results from chronic pancreatitis. The most common cause of chronic pancreatitis is alcohol, although other conditions causing recurrent episodes of acute pancreatitis may also lead to chronic pancreatitis, including hereditary pancreatitis. The diagnosis of chronic pancreatitis is usually supported by evidence of pancreatic atrophy, ductal dilatation or calcification, or a mass/pseudocyst, as detected by one or more imaging procedures (abdominal radiograph, sonogram, CT or ERCP). It is noteworthy that serum amylase, lipase and trypsinogen levels are elevated in acute pancreatitis, but that these tests are usually within or below normal limits in patients with pancreatic insufficiency or chronic pancreatitis.

GENETIC BASIS OF DISEASE The genetics of CF are presented in Chapter 37. Briefly, the inheritance pattern of CF is autosomal recessive, the gene causing $\mathrm{CF}$ was mapped to chromosome VII by linkage analysis, and this gene was identified by positional cloning. The protein product of this gene was named the CF transmembrane conductance regulator (CFTR), based on the anticipated function of the protein. The CFTR gene contains 27 exons, spans $250 \mathrm{~kb}$, and encodes an integral membrane protein containing 1480 amino acids. In many types of epithelial cells, CFTR regulates ion fluxes across the plasma membrane by functioning as a cAMP-regulated $\mathrm{Cl}^{-}$channel and as a regulator of other ion channels.

Much has been learned from mutational analyses of the CFTR gene. A single mutation, deletion of the phenylalanine normally in position $508(\Delta \mathrm{F} 508)$, accounts for roughly $70 \%$ of CF alleles. To date, over 700 additional mutations have been identified in other abnormal CF alleles, of which none account for more than $4 \%$ of $\mathrm{CF}$ alleles. CF patients vary widely with respect to the severity and progression of their pulmonary and pancreatic disease. In the case of CF pulmonary disease, it has been difficult to correlate subsets of CF-causing CFTR mutations with mild vs severe disease. This suggests that factors other than genotype play an important role in determining the progression of CF pulmonary disease. In the case of CF pancreatic disease, by contrast, clear genotype-phenotype correlations do exist. These correlations are so striking that they were recognized as part of the initial identification of the CFTR gene, in which it was noted that almost all $\Delta \mathrm{F} 508$ homozygotes have pancreatic insufficiency. By contrast, pancreatic insufficiency is exceptional in compound heterozygotes with certain less common mutations (e.g., $\triangle \mathrm{F} 508 / \mathrm{R} 117 \mathrm{H})$. Thus, the $C F T R$ genotype is the predominant factor determining the severity of CF pancreatic disease, whereas other factors play a greater role in determining the progression of $\mathrm{CF}$ lung disease.

Apart from CF, Shwachman's syndrome and hereditary pancreatitis are additional examples of inherited diseases in which a primary defect in pancreatic function leads to pancreatic insufficiency. It is not known whether genetic factors predispose to chronic pancreatitis or pancreatic insufficiency associated with other conditions such as alcoholism.

MOLECULAR PATHOPHYSIOLOGY OF DISEASE CF pancreatic disease is associated with characteristic pathologic and 


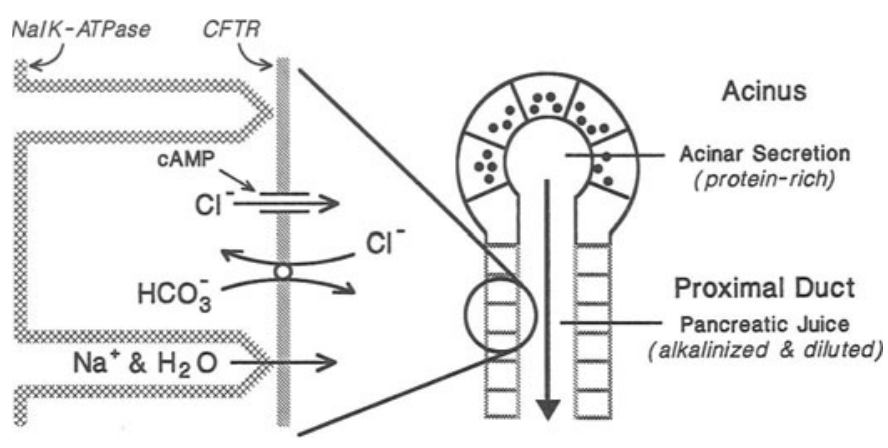

Figure 44-2 A model summarizing the role of the cystic fibrosis transmembrane conductance regulator, CFTR, during exocrine secretion by the pancreas. CFTR normally occurs at the apical membranes of the epithelial cells lining small pancreatic ducts. When activated by cAMP, CFTR allows $\mathrm{CL}^{-}$ions to flow across the apical membrane into the duct lumen. $\mathrm{Cl}^{-}$ions in the duct lumen lead to $\mathrm{HCO}_{3}{ }^{-} / \mathrm{Cl}^{-}$ exchange, and this tends to alkalinize the pancreatic juice. The secreted anions also draw $\mathrm{Na}^{+}$ions and $\mathrm{H}_{2} \mathrm{O}$ into the lumen, thereby diluting the pancreatic juice. In cystic fibrosis, the absence of CFTR causes defective dilution and alkalinization of the pancreatic juice as it passes through the duct, leading eventually to obstruction of the ducts by inspissated secretions. (From Marino CR, Matovcik LM, Gorelick FS, Cohn JA. Localization of the cystic fibrosis transmembrane conductance regulator in pancreas. J Clin Invest 1991;88:712.)

physiologic abnormalities. Obstruction of the intralobular pancreatic ducts is an early pathologic event and impaired pancreatic secretion of bicarbonate is an early physiologic defect. These findings suggest that CFTR may normally function to prevent obstruction of the intralobular ducts and to promote bicarbonate secretion. These concepts are supported by available data concerning the localization of CFTR in human pancreas. The predominant site of CFTR mRNA and protein in pancreas is in the cells lining the proximal ductules. Within these duct epithelial cells, CFTR protein is predominantly detected as a component of the apical plasma membrane.

The distribution of CFTR in pancreas supports the model shown in Fig. 44-2, which postulates an important role for CFTRassociated $\mathrm{Cl}^{-}$channels in the regulation of ductular secretion. According to this model, secretory stimuli act via cAMP to activate CFTR and thereby open $\mathrm{Cl}^{-}$channels at the apical membrane of the duct cell. Because of the driving force provided by the $\mathrm{Na}^{+} / \mathrm{K}^{+}-$ATPase at the basolateral membrane, opening apical $\mathrm{Cl}^{-}$channels results in $\mathrm{Cl}^{-}$efflux across the apical membrane, leading to movement of $\mathrm{Na}^{+}$and water into the lumen and thereby diluting the pancreatic juice. Increased lumenal $\mathrm{Cl}^{-}$also activates $\mathrm{Cl}^{-} / \mathrm{HCO}_{3}{ }^{-}$exchange at the duct-cell apical membrane, and this alkalinizes the pancreatic juice. This model suggests that CFTR in the duct cell contributes to the normal dilution and alkalinization of pancreatic juice. In humans with $\mathrm{CF}$, the ultimate result of the absence of CFTR at this site is a defect in the dilution and alkalinization of the protein-rich acinar secretions in the duct lumen, leading to the formation of protein plugs and thereby to pancreatic injury in $\mathrm{CF}$.

Uncertainty remains concerning the precise mechanism by which pancreatic injury results from defective dilution and alkalinization of the pancreatic juice in CF. Dilution and alkalinization both may help to prevent precipitation of proteins as the pancreatic juice flows through the ducts. Beyond this, data from in vitro models suggest that alkalinization of the lumen of the pancreatic duct system may also be important for maintaining normal acinar cell function during exocytosis.

MANAGEMENT AND THERAPY Pancreatic enzyme replacement is the cornerstone of therapy for pancreatic insufficiency. Widely used enzyme preparations include a combination of lipase, amylase, and proteases. Because lipase is rapidly degraded at low $\mathrm{pH}$, replacement enzymes are commonly administered either as enteric-coated microspheres or in combination with an $\mathrm{H}_{2}$-receptor antagonist. Moderate enzyme doses are usually sufficient to achieve partial correction of steatorrhea with marked improvement in nutrition. Complete correction of steatorrhea is usually not possible even when very high enzyme doses are administered. High enzyme doses should be used with caution because they have been associated with colonic strictures.

Pain control is often the predominant issue in treating chronic pancreatitis. Structural causes for pain include ductal obstruction as a result of strictures, tumor, pseudocyts or stones, and a parenchymal compartment syndrome in which exocrine tissue is encased by fibrosis. Pain resulting from structural causes is typically intermittent and often occurs when serum cholecystokinin levels increase after a fat- or protein-rich meal. Medical therapy for this type of pain can include using gastric acid suppression, or pancreatic enzyme supplements. Narcotics may be added for more severe pain and may also reduce pancreatic exocrine secretion. Surgical options include procedures designed to relieve areas of ductal obstruction or fibrotic encasement of functional parenchyma. Chronic pancreatitis can also cause pain resulting from inflammation. This pain can be severe and unremitting, and surgical resection and celiac ganglion ablation are important management options.

FUTURE DIRECTIONS To date, the genes causing two forms of pancreatic insufficiency have been identified, hereditary pancreatitis and CF. For both diseases, exciting progress has been made in defining the mechanisms leading to pancreatic dysfunction. As these mechanisms become better understood, this knowledge may lead to new strategies for delaying the progression of pancreatic injury. Such strategies could benefit patients with hereditary pancreatitis or $\mathrm{CF}$ and might also be of benefit to patients with exocrine pancreatic injury resulting from other causes. More generally, it is noteworthy that, even though alcohol is the most common cause of chronic pancreatic dysfunction, this condition only develops in a small fraction $(<5 \%)$ of alcoholics. It seems plausible that genetic factors may predispose to pancreatic disease among alcoholics, and testing this hypothesis will be an important goal of future work.

\section{SELECTED REFERENCES}

Durie PR. Inherited and congenital disorders of the exocrine pancreas. Gastroenterologist 1996;4:169-187.

Frey CF. Current management of chronic pancreatitis. Adv Surg 1995;28:337-370.

Gorry MC, Gabbaizedeh D, Furey W, Gates LK Jr., Preston RA, Aston CE, Zhang Y, Ulrich C, Ehrlich GD, Whitcomb DC. Multiple mutations in the cationic trypsinogen gene are associated with hereditary pancreatitis. Gastroenterology 1997;113:1063-1068.

Kingsnorth A. Role of cytokines and their inhibitors in acute pancreatitis. Gut 1997;40:1-4.

Kloppel G, Maillet B. A morphological analysis of 57 resection specimens and 9 autopsy pancreata. Pancreas 1991;6:266-274.

Kusske AM, Rongione AJ, Reber HA. Cytokines and acute pancreatitis [editorial]. Gastroenterology 1996;110:639-642. 
Marino CR, Matovcik LM, Gorelick FS, Cohn JA. Localization of the cystic fibrosis transmembrane conductance regulator in pancreas. J Clin Invest 1991;88:712-716.

Ranson JHC, Rifkind KM, Roses DF, Fink SD, Eng K, Spencer FC. Prognostic signs and the role of operative management in acute pancreatitis. Surg Gynecol Obstet 1974;139:69-81.

Rinderknecht H. Pancreatic secretory enzymes. In: Liang V, Go W, et al., Eds. The Pancreas: Biology, Pathobiology, and Disease, 2nd ed. New York: Raven, 1993; pp. 219-251.
Sainio V, Kemppainen E, Puolakkainen P, et al. Early antibiotic treatment in acute pancreatitis. Lancet 1995;346:663-667.

Slaff J, Jacobson D, Tillman C, Curington C, Toskes P. Protease-specific suppression of pancreatic exocrine secretion. Gastroenterology 1984;87:44-52.

Whitcomb DC, Gorry MC, Preston RA, et al. Hereditary pancreatitis is caused by a mutation in the cationic trypsinogen gene. Nat Genet 1996;13:141-145.

Wyllie R. Hereditary pancreatitis. Am J Gastroenterol 1997;92:1079-1080. 